\title{
Energy-Efficiency Analysis of Regenerative Cooperative Systems Under Spatial Correlation
}

\author{
Mulugeta K. Fikadu ${ }^{1}$, Paschalis C. Sofotasios ${ }^{1,2}$, Mikko Valkama ${ }^{1}$, Qimei Cui ${ }^{3}$, and George K. Karagiannidis ${ }^{2,4}$ \\ ${ }^{1}$ Department of Electronics and Communications Engineering, Tampere University of Technology, 33101 Tampere, Finland. \\ e-mail: \{mulugeta.fikadu; paschalis.sofotasios; mikko.e.valkama\} @tut.fi \\ ${ }^{2}$ Department of Electrical and Computer Engineering, Aristotle University of Thessaloniki, 54124 Thessaloniki, Greece. \\ e-mail: geokarag@auth.gr \\ ${ }^{3}$ Wireless Technology Innovation Institute, Beijing University of Posts and Telecommunications, 100876 Beijing, China. \\ e-mail: cuiqimei@bupt.edu.cn \\ ${ }^{4}$ Department of Electrical and Computer Engineering, Khalifa University, PO Box 127788, Abu Dhabi, UAE.
}

\begin{abstract}
This work is devoted to the error rate and energyefficiency analysis of regenerative cooperative networks in the presence of multipath fading and spatial correlation. To this end, exact analytic expressions are firstly derived for the symbolerror-rate of $M$-ary quadrature amplitude modulation in a dualhop decode-and-forward relay system under spatially correlated Nakagami $-m$ fading channels and maximum ratio combining at the destination. The derived expressions are subsequently employed in quantifying the energy consumption of the considered system, incorporating both transmit energy and the energy consumed by the transceiver circuits. The overall energy consumption is also minimized for certain quality-of-service requirements and it is shown that depending on the degree of spatial correlation, severity of fading, transmission distance, and relay location, a substantial overall energy reduction is sought when compared to conventional direct transmission.
\end{abstract}

\section{INTRODUCTION}

Emerging communication systems are anticipated to provide high-speed data transmission, efficient wireless access, high quality of service (QoS) and satisfactory network coverage with reduced processing time and widespread use of smart phones and other mobile devices. However, the currently witnessed scarcity of the two core fundamental resources, power and bandwidth, constitutes a significant challenge to satisfy these demands while it is known that wireless channel impairments such as multipath fading, shadowing and interference degrade information signals during wireless propagation. Also, most energy constrained devices are typically powered by small batteries where replacement is a rather difficult and costly procedure [1]-[3]. Thus, finding a robust strategy for energy efficient transmission and minimized energy consumption per successfully communicated information bit is essential in effective design and deployment of wireless systems.

Based on this, cooperative communications have been proposed as an alternative solution that improves coverage and performance under severe fading effects [4], [5]. A distinct feature of cooperative communications is that wireless agents share resources, instead of competing for them. In this context, various resource allocation algorithms and techniques have been proposed for improving the energy efficiency of resource constrained wireless networks. Specifically, the authors in [6] analyzed energy-efficient direct transmission adopting higherlevel modulation for short distances, where circuit power is more dominant than transmission power. The authors in [7] addressed the optimal power allocation and throughput transmission strategy for minimizing the total energy consumption required to transmit a given number of bits. Finally, the authors in [8] analyzed realistic scenarios in the context of energy efficient infrastructure-to-vehicle communications.

It is also widely known that fading phenomena constitute a crucial factor of performance degradation [11]-[16] and the references therein. Based on this, numerous investigations addressed the effect of different types of fading conditions on the performance of cooperative communications, see. e.g. [9], [10] and the reference therein. However, the vast majority of these analyses assume statistically independent communication paths, which is a simplistic assumption as in realistic cooperative scenarios the wireless paths may be spatially correlated. Motivated by this, we firstly derive exact analytic expressions for the symbol-error-rate (SER) of a twohop decode-and-forward (DF) relay system under spatially correlated Nakagami $-m$ fading channels for $M$-ary quadrature amplitude Modulation ( $M-\mathrm{QAM})$ using maximum-ratiocombining (MRC). Secondly, we exploit these expressions to provide a comprehensive analysis of energy-efficiency that leads to meaningful insights on the energy consumption per information bit. This demonstrates that a single relay regenerative cooperative network can be a meaningful solution to several low-energy, low-complexity and low-cost wireless applications. Thus, the offered results are anticipated to be particularly useful in future analyses and designs.

\section{System AND ChanNEL Model}

We consider a two-hop DF, half-duplex (HD) cooperative system that consists of a source node $(\mathrm{S})$, a relay node $(\mathrm{R})$ 
and a destination node (D). In phase I, the source broadcasts the signal to both destination and relay nodes; thus, the corresponding received signals can be represented as

$$
y_{S, D}=\sqrt{\frac{P_{S}}{P_{L_{S, D}}}} h_{S, D} x+n_{S, D}
$$

and

$$
y_{S, R}=\sqrt{\frac{P_{S}}{P_{L_{S, R}}}} h_{S, R} x+n_{S, R}
$$

respectively, where $P_{S}$ is the transmit power, $x$ is the transmitted symbol with normalized unit energy in the first transmission phase and $P_{L_{S, D}}$ and $P_{L_{S, R}}$ denote the path loss components in the S-D and S-R paths, respectively. Also, $h_{S, D}$ and $h_{S, R}$ are the complex fading coefficients of the S-D and S$\mathrm{R}$ wireless links, respectively, whereas $n_{S, D}$ and $n_{S, R}$ are the corresponding AWGN terms. If the signal is correctly decoded at the relay, it is forwarded to the destination during phase II with power $\bar{P}_{R}=P_{R}$. Otherwise, the relay does not transmit and remains idle with $\bar{P}_{R}=0$. Based on this, the signal at the destination during phase II can be represented as follows:

$$
y_{R, D}=\sqrt{\frac{\bar{P}_{R}}{P_{L_{R, D}}}} h_{R, D} x+n_{R, D}
$$

where $\bar{P}_{R}$ is the transmit power of the relay, $P_{L_{R, D}}$ is the path loss of the R-D path and $h_{R, D}$ and $n_{R, D}$ denote the corresponding channel coefficients and the AWGN term, respectively. Finally, the destination combines the direct and relayed signals using MRC reception.

\section{SER FOR $M$-QAM MODULATION IN NAKAGAMI- $m$ FADING WITH SPATIAL CORRELATION}

The end-to-end SER for the case of spatially correlated S-D and R-D paths using MRC reception can be determined with the aid of the MGF method and can be expressed according to (4), at the top of the next page [20], [21]. There, $m_{c}=$ $m_{S, D}=m_{R, D}$, denote the corresponding fading parameters, $\Omega_{i, j}$ is the channel variance, $g_{\mathrm{QAM}}=3 / 2(M-1)$ and

$$
F_{\mathrm{QAM}}[v(\theta)]=\frac{4 C}{\pi} \int_{0}^{\pi / 2} v(\theta) \mathrm{d} \theta-\frac{4 C^{2}}{\pi} \int_{0}^{\pi / 4} v(\theta) \mathrm{d} \theta
$$

where $C=(1-1 / \sqrt{M})$ and $\rho$ is the correlation coefficient.

\section{A. Exact SER for the Direct Transmission}

In this subsection, we derive a closed-form expression for the average SER of direct transmission scheme for $M-\mathrm{QAM}$ constellations, which is particularly useful for the subsequent error and energy-efficiency analysis of the considered system.

Theorem 1. For $P_{S}, P_{L_{S, D}}, \Omega_{S, D}, N_{0}, g_{\mathrm{QAM}} \in \mathbb{R}^{+}, M / 2 \in$ $\mathbb{N}$ and $m_{S, D} \geq \frac{1}{2}$, the symbol error rate of an $M-Q A M$ direct transmission scheme over Nakagami- $m$ fading channels can be expressed as follows:

$$
\begin{aligned}
\overline{\operatorname{SER}}_{D}^{D} & =\frac{2(\sqrt{M}-1)}{\sqrt{\pi} M P_{L_{S, D}}^{-1} K^{-m_{S, D}}}\left\{\frac{\Gamma\left(m_{S, D}+\frac{1}{2}\right)}{\Gamma(m+1)}\right. \\
& \times{ }_{2} F_{1}\left(m_{S, D}, \frac{1}{2}, m_{S, D}+1, P_{L_{S, D}} K\right)+\frac{(\sqrt{M}-1)}{2^{-\frac{1}{2}} \sqrt{\pi}} \\
& \left.\times F_{1}\left(\frac{1}{2}, \frac{1}{2}-m_{S, D}, m_{S, D}, \frac{3}{2}, \frac{1}{2}, \frac{P_{L_{S, D}} K}{2}\right)\right\}
\end{aligned}
$$

where ${ }_{2} F_{1}($.$) and F_{1}($.$) denote the Gauss hypergeometric$ function and the Appell hypergeometric function of the first kind, respectively whereas

$$
K=\frac{m_{S, D} N_{0} P_{L_{S, D}}}{m_{S, D} N_{0} P_{L_{S, D}}+P_{S} \Omega_{S, D} g_{\mathrm{QAM}}} .
$$

Proof. The proof is provided in Appendix A.

\section{B. Exact SER for the Cooperative-Transmission}

The derivation of a closed-form expression for the average SER of the considered scenario is subject to analytic evaluation of the following indefinite trigonometric integral.

Lemma 1. For $a, b, m \in \mathbb{R}^{+}$and $2 m-\frac{1}{2} \in \mathbb{N}$, the following closed-form expression is valid,

$$
\begin{aligned}
\mathcal{J}(a, b, m)= & \int \frac{1}{\left(1+\frac{a}{\sin ^{2}(\theta)}+\frac{b}{\sin ^{4}(\theta)}\right)^{m}} \mathrm{~d} \theta \\
= & -\sum_{l=0}^{2 m-\frac{1}{2}}\left(\begin{array}{c}
2 m-\frac{1}{2} \\
l
\end{array}\right)(-1)^{l} \frac{\cos ^{1+2 l}(\theta)}{(1+2 l)} \\
& \times \frac{\left(2 \sin ^{2}(\theta)+\mathcal{D}\right)^{m}\left(2 \sin ^{2}(\theta)+\mathcal{E}\right)^{m}}{(2+\mathcal{D})^{m}(2+\mathcal{E})^{m}} \\
& \times \frac{F_{1}\left(l+\frac{1}{2}, m, m, l+\frac{3}{2}, \frac{2 \cos ^{2}(\theta)}{2+\mathcal{D}}, \frac{2 \cos ^{2}(\theta)}{2+\mathcal{E}}\right)}{\left(\sin ^{4}(\theta)+a \sin ^{2}(\theta)+b\right)^{m}}
\end{aligned}
$$

where

$$
\left\{\begin{array}{l}
\mathcal{D} \\
\mathcal{E}
\end{array}\right\}=a\left\{\begin{array}{l}
- \\
+
\end{array}\right\} \sqrt{a^{2}-4 b} .
$$

Proof. The proof is provided in Appendix B.

The solution in Lemma 1 is generic and can be also useful in future analyses in natural sciences and engineering including applications relating to emerging wireless communications. To this end, it is used in the proof of the following theorem.

Theorem 2. For $\left\{P_{S}, P_{R}, P_{L_{S, D}}, P_{L_{S, R}}, P_{L_{R, D}}, \Omega_{S, D}, \Omega_{S, R}\right.$, $\left.\Omega_{R, D}, N_{0}\right\} \in \mathbb{R}^{+}, M / 2 \in \mathbb{N}, m_{S, D} \geq \frac{1}{2}, m_{S, R} \geq \frac{1}{2}$, $m_{R, D} \geq \frac{1}{2}, m_{S, D}, 2 m_{c}-\frac{1}{2} \in \mathbb{N}$ and $0 \leq \rho<1$, the SER of $M-Q A M$ based DF relaying over spatially correlated Nakagami-m fading channels can be expressed as follows

$$
\begin{aligned}
\overline{\operatorname{SER}}_{D}^{C} & =\left\{\frac{2 \Gamma\left(m_{c}+\frac{1}{2}\right){ }_{2} F_{1}\left(m_{c}, \frac{1}{2}, m_{c}+1, \frac{1}{1+a_{1}}\right)}{\sqrt{\pi} m_{c} ! M(\sqrt{M}-1)^{-1}\left(1+a_{1}\right)^{m_{c}}}\right. \\
& \left.+\frac{2 \sqrt{2} F_{1}\left(\frac{1}{2}, \frac{1}{2}-m_{c}, m_{c}, \frac{3}{2}, \frac{1}{2}, \frac{1}{2+2 a_{1}}\right)}{\pi M(\sqrt{M}-1)^{-2}\left(1+a_{1}\right)^{m_{c}}}\right\}
\end{aligned}
$$




$$
\begin{aligned}
\overline{\mathrm{SER}}_{D}^{C} & =F_{\mathrm{QAM}}\left[\frac{1}{\left(1+\frac{\left(P_{S} / P_{L_{S, D}}\right) \Omega_{S, D} g_{Q A M}}{N_{0} m_{c} \sin ^{2} \theta}\right)^{m_{c}}}\right] F_{\mathrm{QAM}}\left[\frac{1}{\left(1+\frac{\left(P_{S} / P_{L_{S, R}} \Omega_{S, R} g_{\mathrm{QAM}}\right.}{N_{0} m_{S, R} \sin ^{2} \theta}\right)_{S, R}^{m_{S}}}\right] \\
& +F_{\mathrm{QAM}}\left[\frac{1}{\left(1+\frac{\left[\left(P_{S} \Omega_{S, D} / P_{L_{S, D}}\right)+\left(P_{R} \Omega_{R, D} / P_{L_{R, D}}\right)\right] g_{Q A M}}{N_{0} m_{c} \sin ^{2} \theta}+\frac{(1-\rho) P_{S} P_{R} \Omega_{S, D} \Omega_{R, D} g_{\mathrm{QAM}}^{2}}{N_{0}^{2} P_{L_{S, D}} P_{L_{R, D}} m_{c}^{2} \sin ^{4} \theta}\right.}\right] \\
& \times\left\{1-F_{\mathrm{QAM}}\left[\frac{1}{\left(1+\frac{\left(P_{S} / P_{L_{S, R}}\right) \Omega_{S, R} g_{Q A M}}{N_{0} m_{S, R} \sin ^{2} \theta}\right)^{m_{S, R}}}\right]\right\}
\end{aligned}
$$

$$
\begin{aligned}
& \times\left\{\frac{2 \Gamma\left(m_{S, R}+\frac{1}{2}\right){ }_{2} F_{1}\left(m_{S, R}, \frac{1}{2}, m_{S, R}+1, \frac{1}{1+b_{1}}\right)}{\sqrt{\pi} m_{S, R} ! M(\sqrt{M}-1)^{-1}\left(1+b_{1}\right)^{m_{S, R}}}\right. \\
& \left.+\frac{2 \sqrt{2} F_{1}\left(\frac{1}{2}, \frac{1}{2}-m_{S, R}, m_{S, R}, \frac{3}{2}, \frac{1}{2}, \frac{1}{2+2 b_{1}}\right)}{\pi M(\sqrt{M}-1)^{-2}\left(1+b_{1}\right)^{m_{S, R}}}\right\} \\
& +\left\{1-\frac{2 \Gamma\left(m_{S, R}+\frac{1}{2}\right)(\sqrt{M}-1)}{\sqrt{\pi} m_{S, R} ! M}\right. \\
& \times \frac{{ }_{2} F_{1}\left(m_{S, R}, \frac{1}{2}, m_{S, R}+1, \frac{1}{1+b_{1}}\right)}{\left(1+b_{1}\right)^{m_{S, R}}} \\
& \left.-\frac{2 \sqrt{2} F_{1}\left(\frac{1}{2}, \frac{1}{2}-m_{S, R}, m_{S, R}, \frac{3}{2}, \frac{1}{2}, \frac{1}{2+2 b_{1}}\right)}{\pi M(\sqrt{M}-1)^{-2}\left(1+b_{1}\right)^{m_{S, R}}}\right\} \\
& \times\left\{\sum_{l=0}^{2 m_{c}-\frac{1}{2}}\left(\begin{array}{c}
2 m_{c}-\frac{1}{2} \\
l
\end{array}\right) \frac{(-1)^{l} 4(\sqrt{M}-1)^{2} 2^{m_{c}-l-\frac{1}{2}}}{\left[1+2\left(a_{1}+c_{1}\right) 4 a_{1} d_{1}\right]^{m_{c}}}\right. \\
& \times \frac{F_{1}\left(l+\frac{1}{2}, m_{c}, m_{c}, l+\frac{3}{2}, 2 \mathcal{A}, 2 \mathcal{B}\right)}{M \pi(1+2 l)[(1-2 \mathcal{A})(1-2 \mathcal{B})]^{-m_{c}}} \\
& +\sum_{l=0}^{2 m_{c}-\frac{1}{2}}\left(\begin{array}{c}
2 m_{c}-\frac{1}{2} \\
l
\end{array}\right) \frac{(-1)^{l} 4(\sqrt{M}-1)}{\pi M(1+2 l)\left(a_{1} d_{1}\right)^{m_{c}}} \\
& \left.\times \frac{F_{1}\left(l+\frac{1}{2}, m_{c}, m_{c}, l+\frac{3}{2}, \mathcal{A}, \mathcal{B}\right)}{(1-\mathcal{A})^{-m_{c}}(1-\mathcal{B})^{-m_{c}}}\right\}
\end{aligned}
$$

where

$$
\begin{gathered}
a_{1}=\frac{P_{S} \Omega_{S, D} g_{Q A M}}{\left(P_{L_{S, D}} m_{S, D} N_{0}\right)} \\
b_{1}=\frac{P_{S} \Omega_{S, R} g_{Q A M}}{\left(P_{L_{S, R}} N_{0} m_{S, R}\right)}, \\
c_{1}=\frac{P_{R} \Omega_{R, D} g_{Q A M}}{\left(P_{L_{R, D}} N_{0} m_{R, D}\right)}, \\
d_{1}=\frac{(1-\rho) P_{R} \Omega_{R, D} g_{Q A M}}{\left(P_{L_{R, D}} N_{0} m_{R, D}\right)}
\end{gathered}
$$

and

$$
\left\{\begin{array}{l}
\mathcal{A} \\
\mathcal{B}
\end{array}\right\}=\frac{1}{2+a_{1}+c_{1}\left\{\begin{array}{l}
- \\
+
\end{array}\right\} \sqrt{\left(a_{1}+c_{1}\right)^{2}-4 a_{1} d_{1}}}
$$

Proof. The first, second, and fourth terms in (4) can be expressed in closed-form with the aid of Theorem 1. The third term has the same algebraic representation as (8). As a result, a closed-form expression can be deduced by determining the following specific cases in (5)

$\mathcal{J}\left(a, b, m, 0,\left\{\begin{array}{l}\pi / 2 \\ \pi / 4\end{array}\right\}\right)=\int_{0}^{\left\{\begin{array}{l}\pi / 2 \\ \pi / 4\end{array}\right\}} \frac{1}{\left(1+\frac{a}{\sin ^{2}(\theta)}+\frac{b}{\sin ^{4}(\theta)}\right)^{m}} \mathrm{~d} \theta$.

To this effect and after some algebraic manipulations and substituting in (4) yields (10), which completes the proof.

To the best of the authors' knowledge, the offered results have not been reported previously in the open literature.

\section{Power Consumption Model And Energy OPTIMIZATION ANALYSIS}

\section{A. Power Consumption Model}

In this subsection, we quantify the total energy consumption required to transmit $L$ information bits from the source to the destination. We assume that the transceiver circuitry operates on multi-mode basis i.e. $i$ ) when there is a signal to transmit, the circuits are in active mode; $i i$ ) when there is no signal to transmit, the circuits operate on a sleep mode; $i i i$ ) the circuits are in transient mode during the switching process from sleep mode to active mode. To this effect, by denoting the duration of the sleep, transient and active modes as $T_{s p}, T_{t r}$ and $T_{o n}$, respectively, the total energy required to transmit and receive $L$ information bits can be expressed as [6]

$$
E=P_{o n} T_{o n}+P_{s p} T_{s p}+P_{t r} T_{t r}
$$

where $P_{o n}, P_{s p}$ and $P_{t r}$ denote the power consumption values during the active, sleep and transient duration, respectively. The active mode power comprises the transmit power $P_{t}$, power consumption of the RF amplifier $\alpha P_{t}$ and the transmitter and receiver circuit powers of $P_{C T_{x}}$ and $P_{C R_{x}}$, namely,

$$
P_{\text {on }}=(1+\alpha) P_{t}+P_{C T_{x}}+P_{C R_{x}}
$$

where $\alpha=\frac{\xi}{\eta}-1$, with $\eta$ denoting the drain efficiency of the amplifier where for the case of square uncoded $M-\mathrm{QAM}$, $\xi=3 \frac{\sqrt{M}-1}{\sqrt{M}+1}$, [17]. Also, $T_{\text {on }}=\frac{L T_{s}}{b}=\frac{L}{b B}$, where $b=\log _{2} M$ 
is the constellation size and $T_{s}$ is the symbol duration which relates to the bandwidth $B$ as $T_{s} \approx \frac{1}{B}$ [18]. It is noted that in realistic circuit designs $P_{s p} \simeq 0$, while the power consumption during the transient mode refers to the power consumption of the frequency synthesizers. Hence, it follows that $P_{t r}=2 P_{L O}$.

\section{B. Energy Optimization Analysis}

In this subsection, we analyze the total energy required to transmit information efficiently from the source to the destination for direct and cooperative transmissions.

1) Direct Transmission: Using (17) and (18) and recalling that $P_{s p} \simeq 0$ and $P_{t r}=2 P_{L O}$, the average energy consumption per information bit in the direct mode can be given by

$$
\bar{E}_{T}^{D}=\frac{\left((1+\alpha) P_{S}+P_{C T_{x}}+P_{C R_{x}}\right) T_{o n}+2 P_{L O} T_{t r}}{L}
$$

where $P_{S}$ denotes the source transmit power. Based on (19) and for fixed circuit powers with the maximum transmission power and target bit error rate, $p^{*}$, as constraints, the corresponding energy optimization problem can be represented as

$$
\begin{array}{cc} 
& \min _{P_{S}} \bar{E}_{T}^{D} \\
\text { subject to: } & P_{S} \leq P_{\text {maxt }}, \quad P_{S} \geq 0 \\
& \overline{\mathrm{BER}}_{D}^{D}=p^{*} .
\end{array}
$$

Deriving the minimum average energy required in the direct transmission, requires prior computation of the corresponding BER. This is realized with the aid of (6) and the following useful approximations $F_{1}\left(\frac{1}{2} ; \frac{1}{2}-m, m, \frac{3}{2} ; \frac{1}{2}, \frac{1}{2+2 a_{1}}\right) \simeq$ $F_{1}\left(\frac{1}{2} ; \frac{1}{2}-m, m, \frac{3}{2} ; \frac{1}{2}, 0\right)$ and ${ }_{2} F_{1}\left(m, \frac{1}{2} ; m+1 ; \frac{1}{1+a_{1}}\right) \simeq 1$. To this effect, the following accurate closed-form BER approximation for $M-\mathrm{QAM}$ constellations is deduced

$$
\begin{aligned}
\overline{\mathrm{BER}}_{D}^{D} & \simeq \frac{2(\sqrt{M}-1) \Gamma\left(m+\frac{1}{2}\right)}{\sqrt{\pi} M m !\left(1+a_{1}\right)^{m} \log _{2} M} \\
& +\frac{4 F_{1}\left(\frac{1}{2} ; \frac{1}{2}-m, m, \frac{3}{2} ; \frac{1}{2}, 0\right)}{\sqrt{2} \pi\left(1+a_{1}\right)^{m} \log _{2} M}\left(1-\frac{1}{\sqrt{M}}\right)^{2} .
\end{aligned}
$$

Importantly, the Appell function in (21) can be expressed in terms of the Gauss hypergeometric function, namely

$$
F_{1}\left(\frac{1}{2} ; \frac{1}{2}-m, m, \frac{3}{2} ; \frac{1}{2}, 0\right)={ }_{2} F_{1}\left(\frac{1}{2}, \frac{1}{2}-m ; \frac{3}{2}, \frac{1}{2}\right)
$$

and thus, the BER expression in (21) becomes

$$
\begin{aligned}
\overline{\mathrm{BER}}_{D}^{D} & \simeq \frac{2(\sqrt{M}-1) \Gamma\left(m+\frac{1}{2}\right)}{\sqrt{\pi} M m !\left(1+a_{1}\right)^{m} \log _{2} M} \\
& +\frac{4{ }_{2} F_{1}\left(\frac{1}{2}, \frac{1}{2}-m ; \frac{3}{2}, \frac{1}{2}\right)}{\sqrt{2} \pi\left(1+a_{1}\right)^{m} \log _{2} M}\left(1-\frac{1}{\sqrt{M}}\right)^{2} .
\end{aligned}
$$

It is evident that (23) is a function of the modulation order, the severity of multipath fading and $a_{1}$. Therefore, by substituting the targeted $\operatorname{QoS} p^{*}$ in (23), recalling that
$a_{1}=\left(\begin{array}{ll}P_{S} & \Omega_{S, D} \\ g_{Q A M}\end{array}\right) /\left(N_{0} P_{L_{S, D}}\right)$ and carrying out some algebraic manipulations, it follows that

$$
P_{S} \simeq \frac{m N_{0} P_{L_{S, D}}}{\Omega_{S, D} g_{Q A M}}\left[\left(\frac{C}{p^{*}}\right)^{1 / m}-1\right]
$$

where

$$
C=\frac{4(\sqrt{M}-1)}{M \log _{2} M}\left[\frac{{ }_{2} F_{1}\left(\frac{1}{2}, \frac{1}{2}-m ; \frac{3}{2} ; \frac{1}{2}\right)}{\sqrt{2} \pi(\sqrt{M}-1)^{-1}}+\frac{\Gamma\left(m+\frac{1}{2}\right)}{2 \sqrt{\pi} m \Gamma(m)}\right] .
$$

2) Cooperative Transmission: In this case the total average power consumption is given by

$P_{T}^{C}=\left\{\begin{array}{l}P_{C T_{x}}+(1+\alpha) P_{S}+2 P_{C R_{x}}, \text { with } \operatorname{Pr}=1 \\ P_{C T_{x}}+(1+\alpha) P_{R}+P_{C R_{x}}, \text { with } \operatorname{Pr}=1-\overline{\mathrm{SER}}_{S, R}\end{array}\right.$

where $P_{R}$ is the relay transmit power. Using (26) the average power consumption in the cooperative transmission mode is

$$
\begin{aligned}
\bar{P}_{T}^{C} & =P_{C T_{x}}+(1+\alpha) P_{S}+2 P_{C R_{x}} \\
& +\left(P_{C T_{x}}+(1+\alpha) P_{R}+P_{C R_{x}}\right)\left(1-\overline{\mathrm{SER}}_{S, R}\right) .
\end{aligned}
$$

Based on this, the corresponding total average energy consumption per information bit can be expressed as

$$
\bar{E}_{T}^{C}=\frac{\bar{P}_{T}^{C} T_{o n}+2 P_{L O} T_{t r}}{L} .
$$

Evidently, the energy minimization problem with the two optimization variables, $P_{S}$ and $P_{R}$, can be formulated as

$$
\begin{aligned}
& \min _{P_{S}, P_{R}} \bar{E}_{T}^{C}\left(P_{S}, P_{R}\right) \\
\text { subject to: } & \left(P_{S}+P_{R}\right) \leq P_{\text {maxt }}, P_{S} \geq 0, P_{R} \geq 0 \\
& \overline{\operatorname{BER}}_{D}^{C}\left(P_{S}, P_{R}\right)=p^{*} .
\end{aligned}
$$

For the above optimization problem, the optimal powers at the source and relay nodes that minimize the total energy consumption under the given constrains can be evaluated with the aid of numerical optimization techniques such as MATLAB optimization tool box and its fimicon function.

\section{Numerical Results and Analysis}

This section analyzes the average total energy consumption of the radio access system assuming that the S-D and R-D paths are spatially correlated with $\Omega_{S, D}=\Omega_{S, R}=\Omega_{R, D}=$ $0 \mathrm{~dB}$. The path-loss (PL) effects in the system are modeled by the realistic model of $P_{L_{i, j}}[\mathrm{~dB}]=148+40 \log _{10}\left(d_{i, j}[\mathrm{~km}]\right)$, which is also used in device-to-device type communications. Also, the distance relationship satisfies $d_{S, D}=d_{S, R}+d_{R, D}$. The summary of the system parameters used in the analysis is given in Table I where $N_{0}, P_{L O}, T_{t r}$ and $\eta$ are given in [6] whereas the circuit powers are listed in [19].

Fig. 1, illustrates the total energy consumption per information bit as a function of source-to-destination distance, for 4-QAM with fading parameter of $m=1.25$, destination target BER of $10^{-2}$ and zero correlation under the maximum 
TABLE I

SYSTEM PARAMETERS

\begin{tabular}{|c|c|}
\hline \hline$N_{0}=-174 \mathrm{dBm} / \mathrm{Hz}$ & $N_{f}=9 \mathrm{~dB}$ \\
\hline$B=1 \mathrm{MHz}$ & $L=2 \mathrm{kbits}$ \\
\hline$P_{C T_{x}}=98 \mathrm{~mW}$ & $P_{C R_{x}}=112 \mathrm{~mW}$ \\
\hline$P_{L O}=50 \mathrm{~mW}$ & $P_{\operatorname{maxt}}=1500 \mathrm{~mW}$ \\
\hline$\eta=0.35$ & $T_{t r}=5 \mu s$. \\
\hline
\end{tabular}

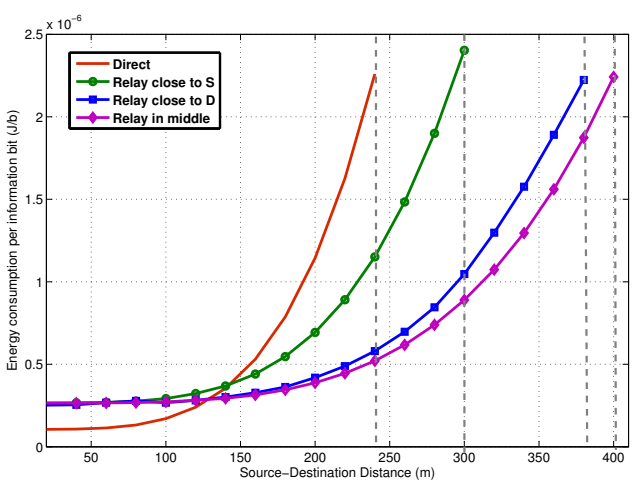

Fig. 1. Energy consumption per information bit versus source-destination distance for relay in different locations over uncorrelated Nakagami-1.25 at target BER of $10^{-2}$ for $4-\mathrm{QAM}$ Modulation.

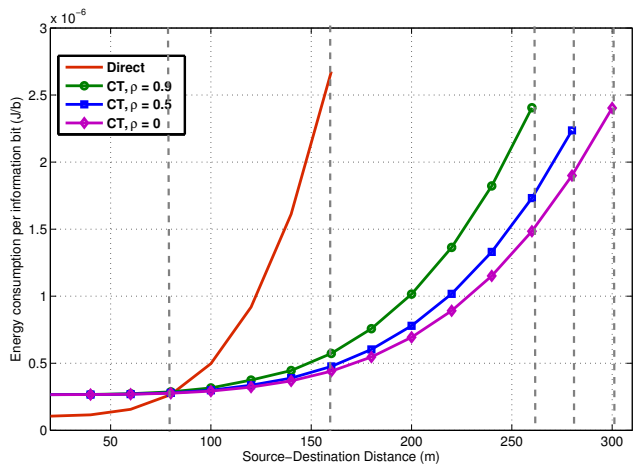

Fig. 2. Energy consumption per information bit versus source-destination distance for relay located in the middle over correlated Nakagami-1.25 at target BER of $10^{-3}$ for $4-\mathrm{QAM}$ Modulation.

transmit power constraint when the relay is located in different locations with $f=d_{S, R} / d_{S, D}$, for the case of optimal power allocation. It is observed that distance thresholds separate the regions where DT performs better than $\mathrm{CT}$ and vice-versa. Furthermore, it is shown that when the relay is located in the middle, i.e. $(f=0.5)$, renders the best energy efficiency among all relay locations. However, it is shown that at relatively small distances (e.g., $0 \leq d_{S, D} \leq 100 \mathrm{~m}$ ), the exact location of the relay does not affect substantially the performance of the cooperative system as it appears to remain almost the same in all considered scenarios.

Fig. 2 depicts the total energy consumption per information bit required for CT and DT as a function of S-D distance over Nakagami-1.25 fading environment at target BER of $10^{-3}$. The relay is placed in the middle and the power is allocated optimally to the source and relay to guarantee minimum energy per information bit. It is observed that at a relatively short distance (e.g., $0 \leq d_{S, D} \leq 80 \mathrm{~m}$ ) the DT scheme outperforms $\mathrm{CT}$, while the system benefits substantially from CT beyond this range. Also, under the given QoS and maximum transmission power constraints, the DT reaches a maximum distance of $160 \mathrm{~m}$ whereas the cooperative system extends beyond this limit even under high spatial correlation conditions. At this threshold transmission distance, the energy savings by $\mathrm{CT}$, defined as $1-\bar{E}^{C} / \bar{E}^{D}$, are $78.6 \%, 82.2 \%$ and $83.5 \%$ for $\rho=0.9, \rho=0.5$ and $\rho=0$, respectively.

\section{CONCLUSIONS}

This work addressed the energy-efficiency analysis and optimization of both direct and regenerative cooperative transmissions in Nakagami $-m$ fading conditions. Novel closed-form expressions were, firstly, derived for the symbol-error rate for $M-\mathrm{QAM}$ modulation, which were subsequently employed in formulating the constrained energy analysis and optimization problems under certain bit-error-rate target and maximum transmit power constraints. The corresponding results indicate that depending on the severity of multipath fading, correlation between the source-destination and relay-destination paths and the location of the relay node, the direct transmission is more energy-efficient than cooperative transmission only for rather short transmission distances and until a certain threshold value. Beyond this distance threshold, the system benefits substantially, as expected, from the cooperation.

\section{APPENDIX}

\section{A. Proof of Theorem 1}

The average SER for the direct transmission is given by

$\overline{\operatorname{SER}}_{D}^{D}=F_{Q A M}\left[\left(1+\frac{\left(P_{S} / P_{L_{S, D}}\right) \Omega_{S, D} g_{Q A M}}{N_{0} m_{S, D} \sin ^{2}(\theta)}\right)^{-m_{S, D}}\right]$.

Evidently, a closed-form expression for (30) is subject to analytic evaluation of the following two integrals

$$
\mathcal{I}\left(a, m ; 0, \frac{\pi}{2}\right)=\int_{0}^{\pi / 2}\left(1+\frac{a}{\sin ^{2}(\theta)}\right)^{-m} \mathrm{~d} \theta
$$

and

$$
\mathcal{I}\left(a, m ; 0, \frac{\pi}{4}\right)=\int_{0}^{\pi / 4}\left(1+\frac{a}{\sin ^{2}(\theta)}\right)^{-m} \mathrm{~d} \theta .
$$

By re-writing the indefinite form of the above integrals as

$$
\mathcal{I}(a, m)=\int \frac{\sin ^{2 m}(\theta)}{\left(\sin ^{2}(\theta)+a\right)^{m}} \mathrm{~d} \theta
$$

and setting $u=\cos ^{2}(\theta)$, one obtains

$$
\mathcal{I}(a, m)=-\int \frac{(1-u)^{m-\frac{1}{2}}}{2 \sqrt{u}(1-u+a)^{m}} \mathrm{~d} u .
$$


The above integral can be expressed in closed-form in terms of the Appell hypergeometric function of the first kind, namely

$\mathcal{I}(a, m)=\frac{-\cos (\theta)}{(1+a)^{m}} F_{1}\left(\frac{1}{2}, \frac{1}{2}-m, m, \frac{3}{2}, \cos ^{2}(\theta), \frac{\cos ^{2}(\theta)}{1+a}\right)$.

Using (35) and the properties of the $F_{1}($.$) function yields$

$$
\mathcal{I}\left(a, m, 0, \frac{\pi}{2}\right)=\frac{\sqrt{\pi} \Gamma\left(m+\frac{1}{2}\right){ }_{2} F_{1}\left(\frac{1}{2}, m, m+1, \frac{1}{1+a}\right)}{2(1+a)^{m} \Gamma(m+1)} .
$$

In the same context, it immediately follows that

$$
\begin{aligned}
\mathcal{I}\left(a, m, 0, \frac{\pi}{4}\right)= & \frac{\sqrt{\pi} \Gamma\left(m+\frac{1}{2}\right){ }_{2} F_{1}\left(\frac{1}{2}, m, m+1, \frac{1}{1+a}\right)}{2(1+a)^{m} \Gamma(m+1)} \\
& -\frac{F_{1}\left(\frac{1}{2}, \frac{1}{2}-m, m, \frac{3}{2}, \frac{1}{2}, \frac{1}{2(1+a)}\right)}{\sqrt{2}(1+a)^{m}} .
\end{aligned}
$$

Therefore, by performing the necessary change of variables in (36) and (37) and substituting in (30) yields (6).

\section{B. Proof of Lemma 1}

The $\mathcal{J}(a, b, m)$ integral can be re-written as follows

$$
\mathcal{J}(a, b, m)=\int \frac{\sin ^{4 m}(\theta)}{\left(\sin ^{4}(\theta)+a \sin ^{2}(\theta)+b\right)^{m}} \mathrm{~d} \theta .
$$

By setting $u=\cos ^{2}(\theta)$ it follows that,

$$
\mathcal{J}(a, b, m)=-\frac{1}{2} \int \frac{(1-u)^{2 m} \mathrm{~d} u}{\sqrt{u} \sqrt{1-u}\left[(1-u)^{2}+a(1-u)+b\right]^{m}} .
$$

Using the binomial theorem in [22, eq. (1.111)] yields

$$
\begin{aligned}
& \mathcal{J}(a, b, m)= \\
& -\sum_{l=0}^{2 m-\frac{1}{2}}\left(\begin{array}{c}
2 m-\frac{1}{2} \\
l
\end{array}\right) \frac{(-1)^{l}}{2} \int \frac{u^{l-\frac{1}{2}} \mathrm{~d} u}{\left[1-u(a+2)+u^{2}+a+b\right]^{m}} .
\end{aligned}
$$

The above integral can be expressed in terms of the Appell function of the first kind and upon performing the necessary counter-substitution and algebraic manipulations yields (8).

\section{ACKNOWLEDGEMENTS}

This work was supported by the Finnish Funding Agency for Technology and Innovation (Tekes) under the project entitled "Energy-Efficient Wireless Networks and Connectivity of Devices-Systems (EWINE-S)", by the Academy of Finland under the projects No. 251138 "Digitally-Enhanced RF for Cognitive Radio Devices and No. 284694 "Fundamentals of Ultra Dense 5G Networks with Application to Machine Type Communication" and by the National Nature Science Foundation of China Project "Grant No. 61471058".

\section{REFERENCES}

[1] G. U. Li, S. Xu, A. Swami, N. Himayat, and G. Fettweis, "Guest-editorial energy-efficient wireless communications," IEEE J. Sel. Areas Commun., vol. 29, no. 8, pp. 1505-1507, Sep. 2011.

[2] D. Feng, C. Jiang, G. Lim, L. J. Cimini, G. Feng, and G.Y. Li, “ A survey of energy-efficient wireless communications," IEEE Commun. Surveys and Tuts., vol. 15, no. 1, pp. 167-178, 1st Quart. 2013.

[3] C. X. Mavromoustakis, G. Kormentzas, G. Mastorakis, A. Bourdena, E. Pallis and C. D. Dimitriou," Joint energy and delay-aware scheme for 5G mobile cognitive radio retworks," in Proc. IEEE Globecom 2014, Dec. 2014, pp. 2624-2630.

[4] Y-W. Hong, W-J. Huang, F-H. Chiu, and C-C. J. Kuo, "Cooperative communications in resource-constrained wireless networks," IEEE Signal Process. Mag., vol. 24, no. 3, pp. 47-57, May 2007.

[5] A. Sendonaris, E. Erkip, and B. Aazhang, "User cooperation diversity part I: System description,” IEEE Trans. Commun., vol. 51, no. 11, pp. 1927-1938, Nov. 2003.

[6] S. Cui, A. Goldsmith, and A. Bahai, "Energy-constrained modulation optimization for coded systems," in Proc. IEEE Globecom 2003, Dec. 2003, pp. 372-376.

[7] R. Devarajan, S. C. Jha, U. Phuyal, and V. K. Bhargava, "Energy-aware user selection and power allocation for cooperative communication system with guaranteed quality-of-service," in Proc. IEEE CWIT '11, May 2011, pp. $216-220$.

[8] T.-D. Nguyen, O. Berder, and O. Sentieys, "Energy-efficient cooperative techniques for infrastructure-to-vehicle communications," IEEE Trans. Intell. Transp. Syst., vol. 12, no. 3, pp. 659-668, Sep. 2011.

[9] D. B. da Costa and S. Aissa, "Capacity analysis of cooperative systems with relay selection in Nakagami-m fading," IEEE Commun. Lett., vol. 13, no. 9, pp. 637-639, Sep. 2009.

[10] T. Q. Duong, G. C. Alexandropoulos, T. A. Tsiftsis, and H. Zepernick, "Outage probability of MIMO AF relay networks over Nakagami-m fading channels," Electr. Lett., vol. 46, no. 17, pp. 1229-1231, Aug. 2010.

[11] M. K. Fikadu, P. C. Sofotasios, M. Valkama, and Q. Cui, "Analytic Performance Evaluation of $M-$ QAM Based Decode-and-Forward Relay Networks over enriched multipath fading channels," in Proc. in WiMob '14, Larnaca, Cyprus, pp. 194-199, Oct. 2014.

[12] K. Ho-Van, P. C. Sofotasios, S. V. Que, T. D. Anh, and T. P. Quang, and L. P. Hong, "Analytic Performance Evaluation of Underlay Relay Cognitive Networks with Channel Estimation Errors,' in IEEE ATC '13, HoChiMinh City, Vietnam, pp. 631-636, Oct. 2013

[13] G. C. Alexandropoulos, P. C. Sofotasios, K. Ho-Van, and S. Freear, "Symbol error probability of DF relay selection over arbitrary Nakagamim fading channels," HINDAWI Journal of Engineering (Invited Paper), Article ID 325045, 2013.

[14] M. K. Fikadu, P. C. Sofotasios, Q. Cui, and M. Valkama, "EnergyOptimized Cooperative Relay Network over Nakagami-m Fading Channels", in IEEE WiMob '13, Lyon, France, pp. 414-421, Oct. 2013.

[15] G. C. Alexandropoulos, A. Papadogiannis, P. C. Sofotasios, "A Comparative Study of Relaying Schemes with Decode-and-Forward over Nakagami-m Fading Channels," Journal of Computer Networks and Communications, (Invited Paper), vol. 2011, Article ID 560528, Dec. 2011.

[16] K. Ho-Van, P. C. Sofotasios, "Bit Error Rate of Underlay Multi-hop Cognitive Networks in the Presence of Multipath Fading," in IEEE ICUFN '13, pp. 620-624, Da Nang, Vietnam, July 2013.

[17] S. Cui, A. J. Goldsmith, A. Bahai, "Energy-constrained modulation optimization," IEEE Trans. Wir. Commun., vol. 4, no. 5, 2349-2360, Sep. 2005.

[18] S. Cui, A. J. Goldsmith, and A. Bahai, "Energy-efficiency of MIMO and cooperative MIMO techniques in sensor networks," IEEE J. Select. Areas Commun., vol. 22, no. 6, pp. 1089-1098, Aug. 2004.

[19] O. Amin, and L. Lampe, "Opportunistic energy efficient cooperative communication," IEEE Wireless Commun. Lett., vol. 1, no. 5, pp. 412-415, Oct. 2012.

[20] Y. Lee and M-H. Tsai, "Performance of decode-and-forward cooperative communications over Nakagami- $m$ fading channels," IEEE Trans. on Veh. Technol., vol. 58, no. 3, pp. 1218-1228, March 2009.

[21] M. K. Simon and M.-S. Alouni, Digital Communication over Fading Channels, 2nd ed., New York: Wiley, 2005.

[22] A. P. Prudnikov, Yu. A. Brychkov, and O. I. Marichev, Integrals and Series, vol.1, Elementary Functions, 1st ed. New York: Academic, 2007. 